https://helda.helsinki.fi

The outcome and timing of death of 17,767 nosocomial

bloodstream infections in acute care hospitals in Finland during 1999-2014

Kontula, Keiju S. K.

2018-05

Kontula , K S K , Skogberg , K , Ollgren , J , Järvinen , A \& Lyytikäinen , O 2018 , ' The outcome and timing of death of 17,767 nosocomial bloodstream infections in acute care hospitals in Finland during 1999-2014 ' , European Journal of Clinical Microbiology \& Infectious Diseases, vol. 37 , no. 5 , pp. 945-952 . https://doi.org/10.1007/s10096-018-3211-0

http://hdl.handle.net/10138/302059

https://doi.org/10.1007/s10096-018-3211-0

publishedVersion

Downloaded from Helda, University of Helsinki institutional repository.

This is an electronic reprint of the original article.

This reprint may differ from the original in pagination and typographic detail.

Please cite the original version. 


\title{
The outcome and timing of death of 17,767 nosocomial bloodstream infections in acute care hospitals in Finland during 1999-2014
}

\author{
Keiju S. K. Kontula ${ }^{1} \cdot$ Kirsi Skogberg ${ }^{1} \cdot$ Jukka Ollgren $^{2} \cdot$ Asko Järvinen $^{1} \cdot$ Outi Lyytikäinen $^{2}$
}

Received: 16 January 2018 / Accepted: 2 February 2018 / Published online: 17 February 2018

(C) Springer-Verlag GmbH Germany, part of Springer Nature 2018

\begin{abstract}
Few studies covering all patient groups and specialties are available regarding the outcome of nosocomial bloodstream infections (BSI). We analyzed the role of patient characteristics and causative pathogens of nosocomial BSIs reported by the hospitals participating in national surveillance in Finland during 1999-2014, in terms of outcome, with particular interest in those leading to death within 2 days (i.e. early death). National nosocomial BSI surveillance was laboratory-based and hospital-wide. Data on nosocomial BSIs was collected by infection control nurses, and dates of death were obtained from the national population registry with linkage to national identity codes. A total of 17,767 nosocomial BSIs were identified; 557 BSIs (3\%) were fatal within 2 days and 1150 (6\%) within 1 week. The 1-month case fatality was 14\% (2460 BSIs), and 23\% of the deaths occurred within 2 days and $47 \%$ within 1 week. The patients who died early were older than those who survived $>28$ days, and their BSIs were more often related to intensive care. Gram-positive bacteria caused over half of the BSIs of patients who survived, whereas gramnegative bacteria, especially Pseudomonas aeruginosa, caused more often BSIs of patients who died early, and fungi BSIs of patients who died within 1 week. A significant portion of patients with nosocomial BSIs died early, which underlines the importance of rapid recognition of BSI. Hospital-wide surveillance data of causative pathogens can be utilized when composing recommendations for empiric antimicrobial treatment in collaboration with clinicians, as well as when promoting infection prevention.
\end{abstract}

Keywords Bloodstream infection $\cdot$ Nosocomial $\cdot$ Early death $\cdot$ Surveillance $\cdot$ Case fatality

\section{Introduction}

Nosocomial bloodstream infections (BSI) constitute a major part of all BSIs and are related to significant mortality and morbidity. The case fatality of nosocomial BSIs has varied, with reported all-cause 30-day mortality between 24 and 28\% [1-3] and in-hospital case fatality around 30\% [4-7].

So far, rather limited surveillance data has been available on the outcome and the timing of death of nosocomial BSIs, as previous studies have mostly focused on a specific causative

Keiju S. K. Kontula

keiju.kontula@hus.fi

1 Division of Infectious Diseases, Inflammation Center, University of Helsinki and Helsinki University Hospital, P.O. Box 340, 00029 Helsinki, Finland

2 Department of Health Security, National Institute for Health and Welfare, P.O. Box 30, 00271 Helsinki, Finland agent of BSI [8-10], or the data has been obtained from a single tertiary care hospital [11] or few intensive care units (ICU) $[12,13]$, thus representing a selected patient population. A significant proportion of BSI-associated deaths have been previously shown to occur early, within 2 days after the first positive blood culture [14-16]. The knowledge of the predisposing factors related to these early deaths is scarce, although it is likely that the appropriateness of the empiric antimicrobial treatment plays an important role in early mortality, as the initial blood culture results typically become available not until the second to third day after taking the specimen.

In this study, we analyzed patients' characteristics and causative pathogens of all nosocomial BSIs in the hospitals participating in the Finnish Hospital Infection Program in Finland during 1999-2014 in terms of outcome and timing of death. To our knowledge, similar data on the timing of death has not been presented previously. The objective of our study was to evaluate the role of different factors for mortality of patients with nosocomial BSI and particularly among patients dying early. 


\section{Materials and methods}

\section{Hospitals}

In Finland (population 5.5 million in 2014), the national healthcare system is publicly funded and is organized into 20 geographically and administratively defined healthcare districts (HD), with populations ranging from about 44,000 to 1.6 million. Fifteen HDs have secondary care hospitals and five also provide tertiary care services. Four acute care hospitals from three HDs started in a surveillance project called Finnish Hospital Infection Program (SIRO) in 1999. Participating hospitals increased from the original 4 by $0-2$ hospitals per year to 18 in 2014: 4/5 tertiary care hospitals, $9 / 15$ secondary care hospitals, and 5/ 30 other acute care hospitals representing altogether 13 HDs. Surveillance was active, prospective, and hospital-wide, covering all patients admitted to departments offering acute care [17].

\section{Case finding and definitions}

The instructions generally advise that blood samples should be obtained for culture if the temperature of a patient is > $38^{\circ} \mathrm{C}$ and/or in case of other symptoms or signs compatible with BSI (e.g. hypothermia, hypotonia). Local infection control nurses in each hospital reviewed regularly the laboratory database for positive blood culture results. The Centers of Disease Control and Prevention definition for nosocomial BSI was used, and only laboratory-confirmed BSIs were included in the study [18]. All BSIs in neonates were regarded as nosocomial infections.

Primary BSI refers to bacteremia or fungemia for which there was no documented focal source, including those resulting from intravenous or arterial catheter infections. Secondary BSI was defined as an infection that developed as a consequence of a documented infection with the same microorganism at another body site. Polymicrobial BSI refers to infections due to more than one microorganism in blood cultures within a 48-h period. Of the underlying conditions and possible risk factors, delivery, newborn status, hematological/ solid malignancy, organ transplantation, and hemodialysis were recorded, as well as the presence of a central venous line. All patients who entered the operating room were recorded as having had surgery. Only patients who were hospitalized for at least $24 \mathrm{~h}$ in ICU before the occurrence of BSI were considered to have been exposed to intensive care. All BSIs that became evident during the intensive care stay or within $48 \mathrm{~h}$ after discharge from ICU were considered to be related to intensive care.

Clinical information and microbiological data were recorded by the local infection control nurses on a standardized caserecord form sent monthly to the National Institute for Health and Welfare and entered into a common database.

\section{Outcome}

The case fatality at 2,7 , and 28 days after the first positive blood culture of a particular patient was obtained from the national population registry by use of national identity codes. All deaths occurring within the first 2 days after the onset of the infection (i.e. after taking the first positive blood culture, day 0 ) were referred to as early deaths.

\section{Microbiological resistance}

Both antibiotic-resistant and intermediately susceptible organisms were considered to be resistant when resistance percentages were calculated for the microorganisms. Extendedspectrum $\beta$-lactamase-producing (ESBL) Escherichia coli and Klebsiella pneumoniae were defined as resistant or intermediately susceptible to third-generation cephalosporins. Multidrug-resistant (MDR) Pseudomonas aeruginosa and Acinetobacter were resistant or intermediately susceptible to carbapenems, and carbapenemase-producing Enterobacteriaceae (CPE) refers to E. coli, K. pneumoniae, and Enterobacter sp. resistant or intermediately susceptible to carbapenems.

\section{Analyses and statistics}

Data was analyzed using IBM SPSS Statistics version 22 and Stata 14. Univariate analysis of categorical variables was done with the $\chi^{2}$ test, using Yates's correction, or Fisher's exact test, as appropriate. The differences in distributions between continuous variables were tested by the Kruskal-Wallis test. Piecewise exponential regression model was used to evaluate the effects of patient characteristics and causative pathogens on the hazard of death within different time points of death (0 $2,3-7,8-28$, and $>28$ days after the positive blood culture) (Stata 14, Texas, USA).

\section{Results}

In the 18 hospitals participating in SIRO, a total of 17,893 nosocomial BSIs were identified during 1999-2014; 126 BSIs $(0.7 \%)$ were excluded because the patients' reported national identity codes were inadequate, leaving 17,767 nosocomial BSIs among 15,784 patients in the study. The median age of the patients was 60 years (range $0-103$ ); $15 \%$ were children $<16$ years, and $59 \%$ of the patients were males. Of the BSIs, $22 \%$ were related to intensive care, and $26 \%$ were among patients with a hematological malignancy and $20 \%$ among those with a solid malignancy. There were 1471 (8\%) BSIs in newborns and $348(2 \%)$ BSIs in patients with admission for obstetric care. Of all BSIs, $27 \%$ were secondary, 
Table 1 Patient characteristics of nosocomial bloodstream infections by timing of death in 18 Finnish hospitals during 1999 2014

\begin{tabular}{|c|c|c|c|c|c|}
\hline Characteristic & $\begin{array}{l}\text { Death within } \\
2 \text { days } \\
n=557\end{array}$ & $\begin{array}{l}\text { Death } \\
\text { within } \\
3-7 \text { days } \\
n=593\end{array}$ & $\begin{array}{l}\text { Death } \\
\text { within } \\
8-28 \text { days } \\
n=1310\end{array}$ & $\begin{array}{l}\text { Alive at day } \\
29 \\
n=15,307\end{array}$ & $p$ value \\
\hline Age in years, median (range) & $69(0-95)$ & $68(0-94)$ & $67(0-97)$ & $59(0-103)$ & $<0.001$ \\
\hline Male gender & $340(61 \%)$ & $370(62 \%)$ & $759(58 \%)$ & $8953(58 \%)$ & 0.159 \\
\hline Intensive care & $209(38 \%)$ & $199(34 \%)$ & $338(26 \%)$ & $3177(21 \%)$ & $<0.001$ \\
\hline Central venous catheter & $203(36 \%)$ & $283(48 \%)$ & $498(38 \%)$ & $5888(38 \%)$ & $<0.001$ \\
\hline Catheter-associated BSI & $152(27 \%)$ & $219(37 \%)$ & $374(29 \%)$ & $4989(33 \%)$ & $<0.001$ \\
\hline Secondary bacteremia & $161(29 \%)$ & $156(26 \%)$ & $383(29 \%)$ & $4160(27 \%)$ & 0.311 \\
\hline Preceding surgery & $189(34 \%)$ & $181(31 \%)$ & $403(31 \%)$ & $4913(32 \%)$ & 0.471 \\
\hline Preceding hospital stay & $88(16 \%)$ & $75(13 \%)$ & $213(16 \%)$ & $3170(21 \%)$ & $<0.001$ \\
\hline $\begin{array}{l}\text { Preceding outpatient invasive } \\
\text { procedure } \\
\text { Malignancy }\end{array}$ & $32(6 \%)$ & $33(6 \%)$ & $93(7 \%)$ & $1436(9 \%)$ & $<0.001$ \\
\hline Hematological & $140(25 \%)$ & $158(27 \%)$ & $326(25 \%)$ & $4063(27 \%)$ & 0.535 \\
\hline Solid & $148(27 \%)$ & $159(27 \%)$ & $410(31 \%)$ & $2804(18 \%)$ & $<0.001$ \\
\hline Chronic hemodialysis & $51(9 \%)$ & $69(12 \%)$ & $136(10 \%)$ & $1255(8 \%)$ & 0.001 \\
\hline Organ transplantation & $13(2 \%)$ & $15(3 \%)$ & $36(3 \%)$ & $410(3 \%)$ & 0.956 \\
\hline Newborn status & $25(4 \%)$ & $20(3 \%)$ & $26(2 \%)$ & $1400(9 \%)$ & $<0.001$ \\
\hline Admission for obstetric care & $1(0.2 \%)$ & $1(0.2 \%)$ & $3(0.3 \%)$ & $343(2 \%)$ & $<0.001$ \\
\hline
\end{tabular}

and the urinary tract (35\%) and surgical wound (20\%) were the most common sources of infection.

Of all BSIs, 57\% were caused by gram-positive bacteria, $27 \%$ by gram-negative bacteria, $4 \%$ by fungi, $2 \%$ by anaerobes, and $9 \%$ were polymicrobial and $1 \%$ was not classified. Coagulase-negative staphylococcus (CNS) was the most common single pathogen (22\% of all BSIs), followed by Staphylococcus aureus (15\%), E. coli (12\%), enterococci (8\%), and P. aeruginosa (4\%). Altogether, 398 (2.2\%) BSIs were caused by MDR microbes. The percentage of BSIs caused by MDR microbes increased from $0.4 \%$ in 1999 to $3.4 \%$ in 2014; mostly, the increase occurred in ESBL E. coli and $K$. pneumoniae (from 0 to $11.3 \%$, of all E. coli and K. pneumoniae BSIs).

Of the BSIs, 557 (3\% of all BSIs) were fatal within 2 days (i.e. early deaths) and 1150 (6\% of all BSIs) within 1 week (Table 1). The 1-month case fatality was 14\% (2460 BSIs), and $23 \%$ of these deaths occurred within 2 days and $47 \%$ within 1 week. The differences in the characteristics of the BSIs according to the timing of death (within 2 days, within 3-7 days, within 8-28 days and alive at day 29) are summarized in Table 1 . The patients who died within 28 days were significantly older than those who survived $>28$ days, and the patients with BSIs leading to early death were the oldest. BSIs related to intensive care were significantly more often noted in patients who died within 28 days than in patients who survived $>28$ days, with the highest occurrence in patients who died early. Among patients who died within 28 days, significantly more solid malignancies were noted than among patients who survived $>28$ days, but no difference was observed in the occurrence of hematological malignancies. Central venous catheters, catheter-associated BSIs, and chronic hemodialysis were most commonly seen in patients who died within 3-7 days. There were more secondary bacteremias in BSIs of patients who died within 2 days than in BSIs of patients who survived $>28$ days. Among patients with secondary bacteremias who died within 2 days, the respiratory tract $(24 \%)$ was the most common source of infection, followed by the gastrointestinal tract $(21 \%)$ and the urinary tract (21\%). BSIs related to preceding hospital stay and outpatient invasive procedure and BSIs related to admission for obstetric care were most commonly observed in patients who survived $>28$ days, as well as BSIs in newborns.

Gram-positive bacteria caused $58 \%$ and gram-negative $27 \%$ of the BSIs of patients who survived $>28$ days, whereas among patients who died early, gram-negative bacteria caused $39 \%$ of the BSIs and gram-positive $36 \%$ (Table 2). The higher occurrence of BSIs caused by gram-positive bacteria among patients who survived $>28$ days was mainly due to the higher proportions of CNS (23\%) and S. aureus (16\%). Of the gramnegative bacteria causing BSIs leading to early death, E. coli (14\%) and P. aeruginosa (12\%) were the most common pathogens. Anaerobic bacteria were isolated in $4 \%$ of the BSIs leading to early death, whereas in $2 \%$ of the BSIs in patients who survived $>28$ days. BSIs caused by fungi and polymicrobial BSIs were significantly more often identified among patients with BSIs who died within 28 days than in patients who survived $>28$ days. Candida albicans was the 
Table 2 Causative pathogens of nosocomial bloodstream infections by timing of death in 18 Finnish hospitals during 1999 2014

\begin{tabular}{|c|c|c|c|c|c|}
\hline Pathogen & $\begin{array}{l}\text { Death within } \\
2 \text { days } \\
n=557\end{array}$ & $\begin{array}{l}\text { Death } \\
\text { within } \\
3-7 \text { days } \\
n=593\end{array}$ & $\begin{array}{l}\text { Death } \\
\text { within } \\
8-28 \text { days } \\
n=1310\end{array}$ & $\begin{array}{l}\text { Alive on day } \\
29 \\
n=15,307\end{array}$ & $p$ value \\
\hline Gram-positive bacteria & $203(36 \%)$ & $293(49 \%)$ & $649(50 \%)$ & $8894(58 \%)$ & $<0.001$ \\
\hline Staphylococcus aureus* & $55(10 \%)$ & $63(11 \%)$ & $192(15 \%)$ & $2382(16 \%)$ & \\
\hline Enterococci* & $52(9 \%)^{\mathrm{a}}$ & $81(14 \%)$ & $155(12 \%)$ & $1123(7 \%)$ & \\
\hline $\begin{array}{l}\text { Coagulase-negative } \\
\text { staphylococci* }\end{array}$ & $51(9 \%)^{b}$ & $109(18 \%)$ & $231(18 \%)$ & $3507(23 \%)$ & \\
\hline Streptococcus pneumoniae & $10(2 \%)$ & $7(1 \%)$ & $3(0.2 \%)$ & $145(1 \%)$ & \\
\hline Streptococcus agalactiae & $8(1 \%)$ & $3(0.5 \%)$ & $4(0.3 \%)$ & $416(3 \%)$ & \\
\hline Other & $27(5 \%)^{\mathrm{c}}$ & $30(5 \%)$ & $64(5 \%)$ & $1321(9 \%)$ & \\
\hline Gram-negative bacteria & $216(39 \%)$ & $135(23 \%)$ & $334(25 \%)$ & $4115(27 \%)$ & $<0.001$ \\
\hline Escherichia coli* & $80(14 \%)$ & $44(7 \%)$ & $115(9 \%)$ & $1980(13 \%)$ & \\
\hline Pseudomonas aeruginosa* & $68(12 \%)$ & $29(5 \%)$ & $75(6 \%)$ & $485(3 \%)$ & \\
\hline Klebsiella sp. & $26(5 \%)$ & $24(4 \%)$ & $54(4 \%)$ & $702(5 \%)$ & \\
\hline Enterobacter sp. & $20(4 \%)$ & $14(2 \%)$ & $38(3 \%)$ & $403(3 \%)$ & \\
\hline Serratia sp. & $9(2 \%)$ & $11(2 \%)$ & $19(1 \%)$ & $158(1 \%)$ & \\
\hline Other & $13(2 \%)^{\mathrm{d}}$ & $13(2 \%)$ & $33(3 \%)$ & $387(3 \%)$ & \\
\hline Anaerobes* & $21(4 \%)$ & $14(2 \%)$ & $36(3 \%)$ & $372(2 \%)$ & 0.223 \\
\hline Fungi* & $44(8 \%)$ & $66(11 \%)$ & $109(8 \%)$ & $516(3 \%)$ & $<0.001$ \\
\hline Candida albicans & $32(6 \%)$ & $50(8 \%)$ & $85(6 \%)$ & $332(2 \%)$ & \\
\hline Non-albicans Candida & $8(1 \%)$ & $15(3 \%)$ & $22(2 \%)$ & $155(1 \%)$ & \\
\hline Other & $4(0.7 \%)^{\mathrm{e}}$ & $1(0.2 \%)$ & $2(0.2 \%)$ & $29(0.2 \%)$ & \\
\hline Polymicrobial* & $73(13 \%)$ & $78(13 \%)$ & $166(13 \%)$ & $1301(8 \%)$ & $<0.001$ \\
\hline
\end{tabular}

a 33/52 Enterococcus faecium, 18/52 Enterococcus faecalis

${ }^{\mathrm{b}}$ 26/51 Staphylococcus epidermidis

${ }^{c} 8 / 27$ viridans streptococci, 5/27 Corynebacterium sp., 3/27 Streptococcus pyogenes

d 3/13 Stenotrophomonas maltofilia, 3/13 other or unidentified Pseudomonas sp.

e $3 / 4$ unidentified Candida sp.

*Included in the piecewise exponential regression model most common causative fungus species in all BSI groups. The proportions of BSIs caused by MDR microbes in total and by MDR $P$. aeruginosa were highest in BSIs leading to early death (Table 3). Instead, the proportions of BSIs caused by methicillin-resistant $S$. aureus (MRSA) and ESBL E. coli or $K$. pneumoniae were similar in all outcome groups.

Patient characteristics, causative pathogens, and all MDR microbes together listed in Tables 1, 2, and 3 were included in the model, and the effect of age to the hazard for death was presented by a 10 years increase in age. The hazard ratio associated with intensive care admission was highest 3.6 in BSIs leading to death within 0-2 days and decreased systematically thereafter to 0.85 in BSIs leading to death after 28 days (Fig. 1). Among the BSIs leading to death after 28 days, the highest hazards for death were noted in BSIs of patients with solid and hematological malignancy and chronic hemodialysis. The highest hazard for death concerning the causative pathogens of BSIs leading to death within 0-2 days was among BSIs caused by $P$. aeruginosa, whereas in the BSIs leading to death within 3-7 and 8-28 days, the highest were noted among BSIs caused by fungi, enterococci, and polymicrobial BSIs. On the other hand, the hazards for death associated with BSIs caused by MDR pathogens were similar in all outcome groups of BSIs. In the model, the hazard ratios are multiplicative, e.g., for a patient with a BSI related to intensive care and caused by $P$. aeruginosa, the risk of dying within 2 days was ten times higher compared to a patient with BSI not related to intensive care and not caused by $P$. aeruginosa $(3.6 \times 2.7=9.72)$, if their ages were within 10 -year interval.

\section{Discussion}

In our study based on hospital-wide surveillance data of nearly 18,000 nosocomial BSIs, the 1-month case fatality was $14 \%$, and $23 \%$ of the deaths occurred within 2 days (2-day fatality, $3 \%$ ) and $47 \%$ within 1 week (1-week fatality, $7 \%$ ), after taking the first blood culture specimen. We were able to accurately determine the all-cause mortality within the three time points 
Table 3 Causative multidrug-resistant pathogens of nosocomial bloodstream infections by timing of death in 18 Finnish hospitals during 1999-2014

\begin{tabular}{|c|c|c|c|c|}
\hline Multidrug-resistant microbe & $\begin{array}{l}\text { Death within } \\
2 \text { days } \\
n=557\end{array}$ & $\begin{array}{l}\text { Death } \\
\text { within } \\
3-7 \text { days } \\
n=593\end{array}$ & $\begin{array}{l}\text { Death } \\
\text { within } \\
8-28 \text { days } \\
n=1310\end{array}$ & $\begin{array}{l}\text { Alive at day } \\
29 \\
n=15,307\end{array}$ \\
\hline Methicillin-resistant Staphylococcus aureus (MRSA) (\% of all BSIs) & $3(0.5 \%)$ & $1(0.2 \%)$ & $12(0.9 \%)$ & $78(0.5 \%)$ \\
\hline $\begin{array}{l}\text { Extended-spectrum } \beta \text {-lactamase-producing (ESBL) Escherichia coli or Klebsiella } \\
\text { pneumoniae (\% of all BSIs) }\end{array}$ & $10(1.8 \%)$ & $11(1.9 \%)$ & $18(1.4 \%)$ & $212(1.4 \%)$ \\
\hline Multidrug-resistant (MDR) Pseudomonas aeruginosa (\% of all BSIs) & $8(1.4 \%)$ & $3(0.5 \%)$ & $8(0.6 \%)$ & $20(0.1 \%)$ \\
\hline Vancomycin-resistant Enterococcus (VRE) (\% of all BSIs) & 0 & $1(0.2 \%)$ & 0 & $6(0.04 \%)$ \\
\hline Multidrug-resistant (MDR) Acinetobacter (\% of all BSIs) & 0 & $2(0.3 \%)$ & 0 & $5(0.03 \%)$ \\
\hline Carbapenemase-producing Enterobacteriaceae (CPE) (\% of all BSIs) & 0 & 0 & 0 & 0 \\
\hline Total of multi-resistant microbes* (\% of all BSIs) & $21(3.8 \%)$ & $18(3.0 \%)$ & $38(2.9 \%)$ & $321(2.1 \%)$ \\
\hline
\end{tabular}

*Included in the piecewise exponential regression model

by using data from the national population registry. Gramnegative bacteria, especially $P$. aeruginosa, more often caused BSIs of patients who died early than BSIs of patients who survived $>28$ days. On the other hand, BSIs caused by fungi were most common among patients who died within 1 week, and highest hazards for death after 28 days were noted in BSIs
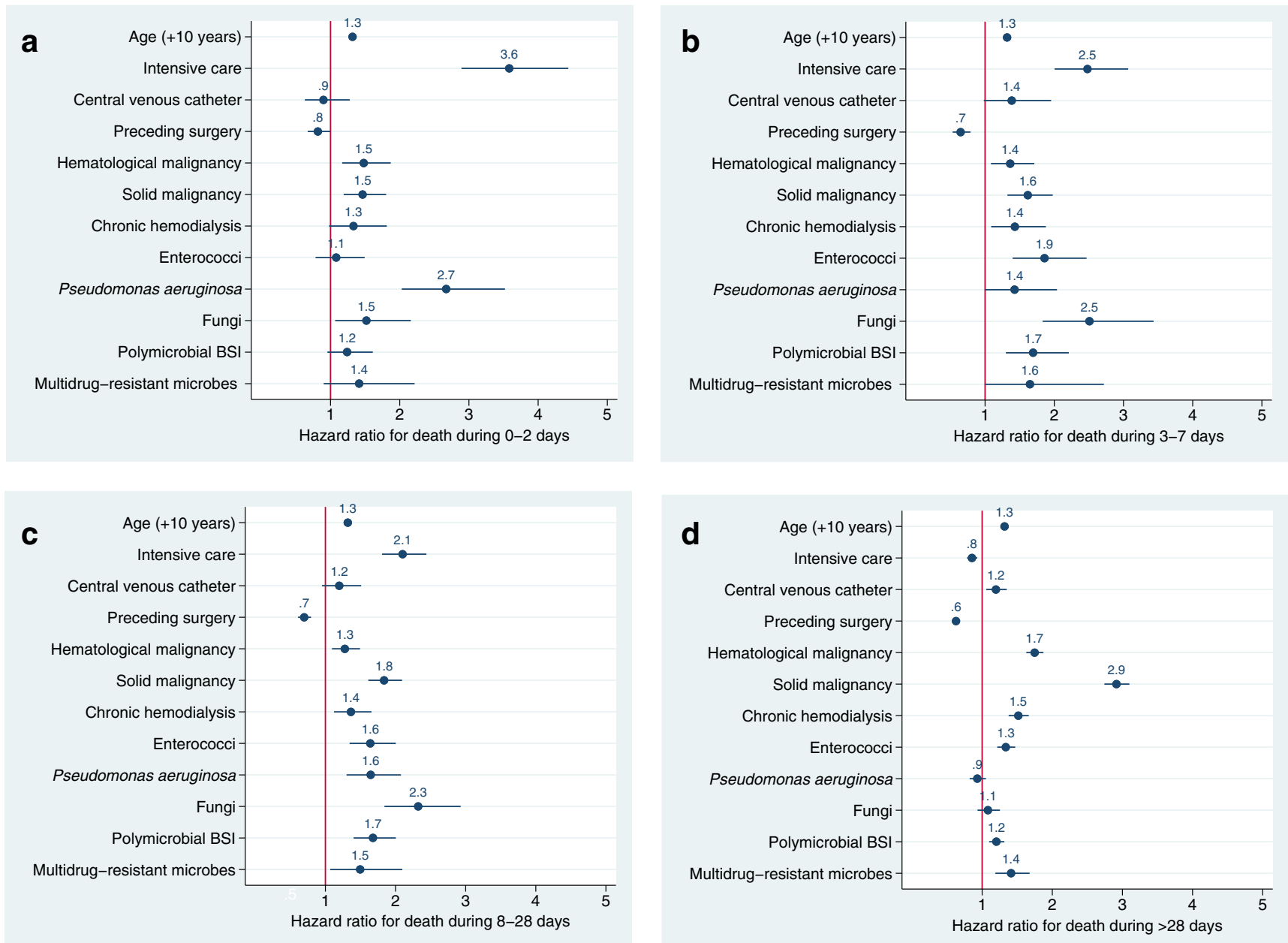

Fig. 1 Hazard ratios for death of nosocomial bloodstream infections according to patient characteristics and causative pathogens in 18 Finnish hospitals during 1999-2014: death during 0-2 days (a), during 3-7 days (b), during 8-28 days (c), and during $>28$ days (d) 
of patients with malignancies. Patients with nosocomial BSIs who died early were older, and these BSIs were more often related to intensive care.

In the present study, the 1-month case fatality (14\%) was lower than in recent studies of nosocomial BSIs from North America and Europe (24-28\%) [1-3]. The case fatality of nosocomial BSIs varies quite markedly between studies depending on how it is reported (1-month, in-hospital mortality), and it is also affected by the differences in the study population, for example whether newborns and ICUs are included in the study, and in the resistance profiles of microbes in the setting. In-hospital mortality can be influenced by the length of stay and differences in the healthcare delivery system.

In previous studies of all BSIs, a notable portion of BSIassociated deaths have been shown to occur early, within 2 days after the first positive blood culture. In a prospective survey from Denmark, nearly $5 \%$ of patients with BSI died before the notification of positive blood cultures [14]. Also, in a population-based study of BSIs in Finland during 2004 2007 , the 30-day case fatality was $13 \%$, and one third of the deaths happened within 2 days after the first blood culture was taken [16]. So far, only limited data has been available on the timing of death of specifically nosocomial BSIs. In the present study, $23 \%$ of the deaths occurred early (3\% of all BSIs) which is slightly less than in studies including all BSIs. One reason for this may be that treatment delays are more common among patients with community-acquired BSIs, as shown in our previous study of BSIs leading to early death [19].

The distributions of gram-positive and gram-negative bacteria and the most commonly seen single pathogens of our total study population were similar to those of recent studies of nosocomial BSIs [20-22]. In our study, some significant differences were observed in the portions of causative pathogens of the BSIs depending on the timing of death of the patients. Gram-negative bacteria, especially $P$. aeruginosa, more often caused BSIs of patients who died early than BSIs of patients in other outcome groups. BSIs caused by fungi were more common among patients who died within 28 days than among those who survived $>28$ days, especially among patients who died within 3-7 days, and the finding was also confirmed by our regression model. In our former study of all BSIs from Finland, the causative pathogens of healthcareassociated BSIs leading to early death were similar: Gramnegative bacteria were most often the causative pathogens (53\%) and P. aeruginosa was the single most common causative agent [19]. Moreover, half of the patients with HA-BSIs who died early received inappropriate empiric antimicrobial treatment, and $41 \%$ of the inappropriately treated patients had a BSI caused by $P$. aeruginosa. Other previous reports have also shown that a notable proportion of patients with nosocomial BSIs receive ineffective initial antimicrobial therapy $[1$, $5,6]$ and that inappropriate initial antimicrobial treatment of BSI caused by $P$. aeruginosa is associated with greater mortality compared to appropriate treatment [23]. Early and effective antimicrobial therapy has been shown to reduce mortality rates in patients with BSIs [24], which underlines the importance of early recognition of the BSI and its healthcare origin and adjustment of the empiric antimicrobial treatment accordingly. Mortality to BSIs caused by fungi may reflect, besides the severity of underlying conditions, the lack of antifungal therapy or prophylaxis, as shown in a previous Finnish study of candidemias during 2004-2007 [25].

Interestingly, in our piecewise exponential regression model, the hazard ratios for death associated with BSIs caused by MDR pathogens were on the same level (1.4-1.6) in all outcome groups. However, despite the increasing trend, only a small proportion of all nosocomial BSIs in our study were caused by MDR microbes (2.2\%) with the highest proportion $(3.8 \%)$ in BSIs leading to early death.

Some differences were also observed in the sources of secondary bacteremias of patients with nosocomial BSI depending on the timing of death. The respiratory tract was the most common source of infection among patients with BSIs leading to early death, followed by the gastrointestinal tract and the urinary tract, whereas among all patients regardless of the outcome, the urinary tract and surgical wound were the most common sources of infection of secondary bacteremias. Similar results were obtained in our previous study of all BSIs leading to early death: The respiratory tract was the most common site of infection, followed by the abdomen and the urinary tract [19]. On the other hand, in studies including only nosocomial BSIs, regardless of the outcome, intravascular device and the urinary tract have been the most common sources of infection $[1,26]$.

We noted some significant differences in the underlying conditions of the patients with nosocomial BSIs depending on the outcome. Patients with BSIs who died early were older, and these BSIs were more often related to ICU stay. Catheter-associated BSIs and chronic hemodialysis were most often perceived among patients with BSIs leading to death within 3-7 days, whereas solid malignancies in patients with BSIs leading to death within 8-28 days. In our previous population-based study from the Helsinki metropolitan area of all BSIs leading to early death, we observed that the patients who died early were significantly older than the survivors, and that one third of these patients had a rapidly fatal underlying disease or a prior do not resuscitate order (DNR) indicating a poor overall condition, as expected [19].

We acknowledge that there are some limitations in this study. First, since the study was based on surveillance data, we did not have information on the patients' specific underlying diseases and the severity of their medical conditions, or information on a possible DNR order of the patients, which certainly have influenced the outcome of the patients. Second, we did not have data on whether the antimicrobial therapy was 
appropriate or not, nor data on delays in the commencement of the treatment. However, as the proportions of BSIs caused by MDR microbes have been low during the study period, these microbes cannot be interpreted as a reason for severe outcome of the patients in terms of inappropriate empiric antimicrobial therapy. Rather, it is probable that the reason for initial ineffective antimicrobial treatment is at least partly due to the intrinsic resistance of the causative pathogens, such as P. aeruginosa and Enterobacter sp. or due to the lack of antifungal treatment. Also, it should be noted that we did not perform subgroup analyses for different Candida sp. concerning the outcome and timing of death. Third, we did not have information on the main cause of death of the patients, but it is likely that among the patients who died early, the BSI was at least a contributing factor.

Our study covered nearly 18,000 nosocomial BSIs during 16 consecutive years in all types of acute care hospitals in different geographical areas of Finland. A notable portion of deaths caused by nosocomial BSIs occurred early, within 2 days. The patients who died early were older than the survivors, and these BSIs were more often caused by gram-negative bacteria, especially $P$. aeruginosa, and were associated with ICU stay. It is likely that at least some of these early deaths would not have been preventable due to severe underlying condition of the patient. Early identification of BSI and its healthcare origin, as well as prompt initiation of appropriate antimicrobial treatment, are essential in prevention of fatal outcome of BSIs. The BSIs in our study represented the causative pathogens of a particular hospital participating in the surveillance project, thus providing with valuable data when promoting infection prevention and when in collaboration with clinicians composing recommendations for empiric antimicrobial treatment among different patient groups. In order to reduce the risk of nosocomial BSIs during hospital stay, implementation of infection control guidelines is highlighted.

Funding K.K. received a grant from the state funding for the Finnish University Hospitals (grant number Y1209TUTKK).

\section{Compliance with ethical standards}

Conflict of interest The authors declare that they have no conflict of interest.

Ethical approval All procedures performed in studies involving human participants were in accordance with the ethical standards of the institutional and/or national research committee and with the 1964 Helsinki declaration and its later amendments or comparable ethical standards. For this type of study, formal consent is not required. This article does not contain any studies with animals performed by any of the authors.

Data availability All data analyzed during the present study are included in this article or are available from the corresponding author on reasonable request.

\section{References}

1. Rodríguez-Baño J, López-Prieto MD, Portillo MM, Retamar P, Natera C, Nuño E et al (2010) Epidemiology and clinical features of community-acquired, healthcare-associated and nosocomial bloodstream infections in tertiary-care and community hospitals. Clin Microbiol Infect 16:1408-1413

2. Søgaard M, Nørgaard M, Dethlefsen C, Schønheyder HC (2011) Temporal changes in the incidence and 30-day mortality associated with bacteremia in hospitalized patients from 1992 through 2006: a population-based cohort study. Clin Infect Dis 52:61-69

3. Lenz R, Leal JR, Church DL, Gregson DB, Ross T, Laupland KB (2012) The distinct category of healthcare associated bloodstream infections. BMC Infect Dis 12:85

4. Wisplinghoff H, Bischoff T, Tallent SM, Seifert H, Wenzel RP, Edmond MB (2004) Nosocomial bloodstream infections in US hospitals: analysis of 24,179 cases from a prospective nationwide surveillance study. Clin Infect Dis 39:309-317

5. McDonald JR, Friedman ND, Stout JE, Sexton DJ, Kaye KS (2005) Risk factors for ineffective therapy in patients with bloodstream infection. Arch Intern Med 165:308-313

6. Mitt P, Adamson V, Lõivukene K, Lang K, Telling K, Päro K et al (2009) Epidemiology of nosocomial bloodstream infections in Estonia. J Hosp Infect 71:365-370

7. Vrijens F, Hulstaert F, Van de Sande S, Devriese S, Morales I, Parmentier Y (2010) Hospital-acquired, laboratory-confirmed bloodstream infections: linking national surveillance data to clinical and financial hospital data to estimate increased length of stay and healthcare costs. J Hosp Infect 75:158-162

8. Lyytikäinen O, Klemets P, Ruutu P, Kaijalainen T, Rantala M, Ollgren J et al (2007) Defining the population-based burden of nosocomial pneumococcal bacteremia. Arch Intern Med 167: $1635-1640$

9. Rodríguez-Baño J, Picón E, Gijón P, Hernández JR, Cisneros JM, Peña $C$ et al (2010) Risk factors and prognosis of nosocomial bloodstream infections caused by extended-spectrum-betalactamase-producing Escherichia coli. J Clin Microbiol 48:17261731

10. Barrasa-Villar JI, Aibar-Remón C, Prieto-Andrés P, Mareca-Doñate R, Moliner-Lahoz J (2017) Impact on morbidity, mortality, and length of stay of hospital-acquired infections by resistant microorganisms. Clin Infect Dis 65:644-652

11. Åttman E, Aittoniemi J, Sinisalo M, Vuento R, Lyytikäinen $\mathrm{O}$, Kärki T et al (2015) Etiology, clinical course and outcome of healthcare-associated bloodstream infections in patients with hematological malignancies: a retrospective study of 350 patients in a Finnish tertiary care hospital. Leuk Lymphoma 56:3370-3377

12. Laupland KB, Gregson DB, Zygun DA, Doig CJ, Mortis G, Church DL (2004) Severe bloodstream infections: a population-based assessment. Crit Care Med 32:992-997

13. Lambert ML, Suetens C, Savey A, Palomar M, Hiesmayr M, Morales I et al (2011) Clinical outcomes of health-care-associated infections and antimicrobial resistance in patients admitted to European intensive-care units: a cohort study. Lancet Infect Dis $11: 30-38$

14. Pedersen G, Schønheyder HC (1997) Patients with bacteremia dying before notification of positive blood cultures: a 3-year clinical study. Scand J Infect Dis 29:169-173

15. Laupland KB, Svenson LW, Gregson DB, Church DL (2011) Longterm mortality associated with community-onset bloodstream infection. Infection 39:405-410

16. Skogberg K, Lyytikäinen O, Ollgren J, Nuorti JP, Ruutu P (2012) Population-based burden of bloodstream infections in Finland. Clin Microbiol Infect 18:E170-E176 
17. Lyytikäinen O, Lumio J, Sarkkinen H, Kolho E, Kostiala A, Ruutu $P$ et al (2002) Nosocomial bloodstream infections in Finnish hospitals during 1999-2000. Clin Infect Dis 35:e14-e19

18. Garner JS, Jarvis WR, Emori TG, Horan TC, Hughes JM (1988) CDC definitions for nosocomial infections, 1988. Am J Infect Control 16:128-140

19. Kontula KS, Skogberg K, Ollgren J, Järvinen A, Lyytikäinen O (2016) Early deaths in bloodstream infections: a population-based case series. Infect Dis (Lond) 48:379-385

20. Edmond MB, Wallace SE, McClish DK, Pfaller MA, Jones RN, Wenzel RP (1999) Nosocomial bloodstream infections in United States hospitals: a three-year analysis. Clin Infect Dis 29:239-244

21. Diekema DJ, Beekmann SE, Chapin KC, Morel KA, Munson E, Doern GV (2003) Epidemiology and outcome of nosocomial and community-onset bloodstream infection. J Clin Microbiol 41: $3655-3660$
22. Hoenigl M, Wagner J, Raggam RB, Prueller F, Prattes J, Eigl S et al (2014) Characteristics of hospital-acquired and community-onset blood stream infections, South-East Austria. PLoS One 9:e104702

23. Micek ST, Lloyd AE, Ritchie DJ, Reichley RM, Fraser VJ, Kollef MH (2005) Pseudomonas aeruginosa bloodstream infection: importance of appropriate initial antimicrobial treatment. Antimicrob Agents Chemother 49:1306-1311

24. Leibovici L, Shraga I, Drucker M, Konigsberger H, Samra Z, Pitlik SD (1998) The benefit of appropriate empirical antibiotic treatment in patients with bloodstream infection. J Intern Med 244:379-386

25. Poikonen E, Lyytikäinen O, Anttila VJ, Koivula I, Lumio J, Kotilainen $\mathrm{P}$ et al (2010) Secular trend in candidemia and the use of fluconazole in Finland, 2004-2007. BMC Infect Dis 10:312

26. Vallés J, Calbo E, Anoro E, Fontanals D, Xercavins M, Espejo E et al (2008) Bloodstream infections in adults: importance of healthcare-associated infections. J Inf Secur 56:27-34 